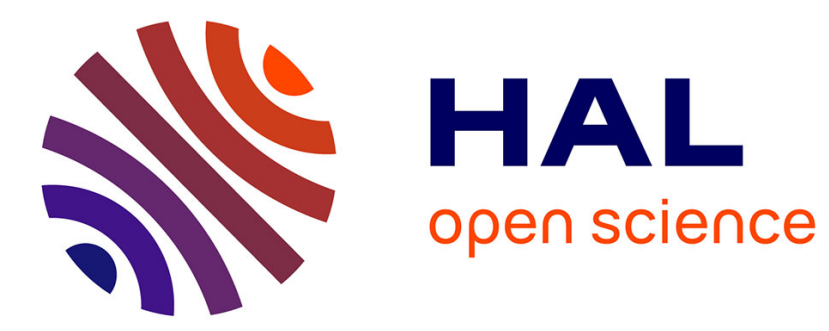

\title{
Sedimentation of a sphere in a fluid channel
}

Olivier Pitois, Ch. Fritz, L. Pasol, M. Vignes-Adler

\section{To cite this version:}

Olivier Pitois, Ch. Fritz, L. Pasol, M. Vignes-Adler. Sedimentation of a sphere in a fluid channel. Physics of Fluids, 2009, 21, pp.103304. 10.1063/1.3253408 . hal-00507437

\section{HAL Id: hal-00507437 https://hal.science/hal-00507437}

Submitted on 10 Sep 2015

HAL is a multi-disciplinary open access archive for the deposit and dissemination of scientific research documents, whether they are published or not. The documents may come from teaching and research institutions in France or abroad, or from public or private research centers.
L'archive ouverte pluridisciplinaire HAL, est destinée au dépôt et à la diffusion de documents scientifiques de niveau recherche, publiés ou non, émanant des établissements d'enseignement et de recherche français ou étrangers, des laboratoires publics ou privés. 


\title{
Sedimentation of a sphere in a fluid channel
}

\author{
Olivier Pitois, ${ }^{\text {a) }}$ Christelle Fritz, Laurentiu Pasol, ${ }^{\text {b) }}$ and Michèle Vignes-Adler \\ Laboratoire de Physique des Matériaux Divisés et des Interfaces, Université Paris-Est, \\ UMR CNRS 8108, 5 Blvd. Descartes, 77454 Marne la Vallée Cedex 2, France
}

(Received 11 March 2009; accepted 21 September 2009; published online 26 October 2009)

\begin{abstract}
We studied both experimentally and numerically the sedimentation velocity of small solid particles through liquid channels merging at the intersection of three soap films. The wall mobility induces a nontrivial behavior for the particle drag coefficient, providing particular transport properties that are not observed for channels with rigid walls. It is shown that for sufficiently small particles, slow and fast motions are observed for the particle along the channel, depending on the particle position within the channel cross section and the sphere/channel size ratio. The velocity corresponding to fast motions can be as high as twice the Stokes velocity in an unbounded fluid. Moreover, the fast motions are not observed anymore when the size ratio exceeds a critical value, which has been found to be approximately equal to 0.5 . As another major difference with the solid wall channel, the sphere velocity does not vanish when the size ratio reaches unity. Instead, the smallest value is found to be $\frac{1}{4}$ of the Stokes velocity. @ 2009 American Institute of Physics. [doi:10.1063/1.3253408]
\end{abstract}

\section{INTRODUCTION}

Stokes law provides the drag force on a solid particle falling at low Reynolds number in an unbounded fluid: ${ }^{1,2}$ $F=3 \pi \mu d V$, where $V$ is the sphere velocity, $d$ is the particle diameter, and $\mu$ is the shear viscosity of the bulk. It is well known that the particle velocity can be drastically modified by the presence of a confining wall. Expressions for the resistance coefficients have been derived for a solid sphere translating parallel to a solid plane. ${ }^{3}$ This is the consequence of the no-slip boundary condition for the fluid flow at the wall. Results have also been obtained as a sphere moves between two plane walls ${ }^{4,5}$ or through a channel. ${ }^{6,7}$ In the reference case of a sphere in settling motion along the axis of a vertical tube, the channel wall is known to exert an additional retardation effect arising from the upward motion of the fluid through the gap between the sphere and the wall. Obviously, considering a more general situation, boundary conditions are likely to differ from this reference, and one can expect some deviations from this old and well established result. For example, slip or apparent slip has been reported for flows over hydrophobic surfaces ${ }^{8,9}$ due to the presence of trapped liquid/gas interfaces. Moreover, wall mobility can result from the fluid nature of the wall itself. ${ }^{10-12}$ The motion of particles in such confined geometries is of great interest and it is relevant to microfluidic applications. ${ }^{13}$ The problem of particle motion through the liquid channels formed between the bubbles of a froth is moreover a major element in the modeling of the froth flotation process of mineral ores. ${ }^{14}$

In the present paper, we report the first observation of the settling motion of a sphere through a channel with fluid interfaces. As shown below, the antagonistic effects of the

\footnotetext{
a) Author to whom correspondence should be addressed. Electronic mail: pitois@univ-mlv.fr.

b) Present address: Laboratoire Physique Thermique-ESPCI, 10 rue Vauquelin, 75231 Paris Cedex 05, France.
}

interface mobility and the liquid backflow give rise to interesting features for the motion of particles in confined geometries.

\section{EXPERIMENT}

The channel is obtained by withdrawing a dedicated frame from a reservoir containing a solution of sodium dodecyl sulfate (SDS) at a concentration $\mathrm{C}=3 \mathrm{~g} / \mathrm{L}$ [see Fig. 1(a)]. The frame consists of a vertical metallic tube on which three rods (1 $\mathrm{mm}$ in diameter) are arranged as a tripod. Due to capillary forces, the cross section of the resulting vertical liquid channel (also called Plateau border) is almost an equilateral triangle except that the three segments joining the vertices are circular arcs of radius $R$ [see Figs. 1(b) and 2(a)]. Each vertex is connected to a thin vertical liquid film —of negligible thickness - attached to the holder and stabilized with the surfactants. The resulting SDS interface is known to exhibit extremely low values of surface viscosity $\left.\left(\sim 10^{-5} \mathrm{~g} / \mathrm{s}\right)\right)^{11}$ The frame is accurately positioned above the reservoir to easily adjust the length of the channel (typical lengths are in the range of 5-15 mm). After generation, the channel and the adjoining films are allowed to freely drain until the system reaches its static equilibrium (this can be verified by imaging the interference fringe pattern in the films). A closed cell covers the above setup ensuring the water vapor saturation of the chamber surrounding the channel. In this manner, liquid flow resulting from evaporation is avoided and channels were found to be stable for hours or more. The inner diameter of the holder is $10 \mathrm{~mm}$ and a circular outlet of $1 \mathrm{~mm}$ in diameter is opened at its lower part: it is used to deliver the particles through the channel. Particles are calibrated glass beads (Duke Scientific Corporation) with density $\rho_{S}=2.45$ and diameter $d=30 \pm 2.1,52.6 \pm 3.2,72.6 \pm 4.4,90.3 \pm 4.5,120 \pm 6$, and $140 \pm 7 \mu \mathrm{m}$. A dedicated delivery system was developed to introduce the particles into the channel without additional 


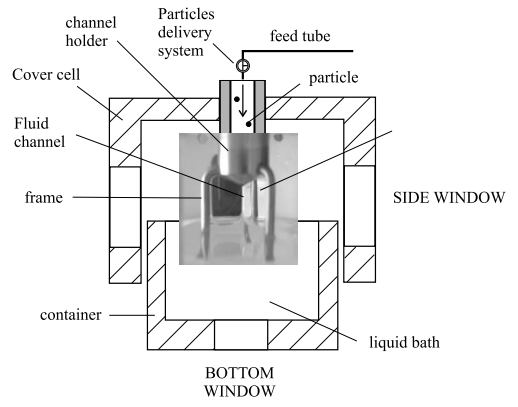

(a)

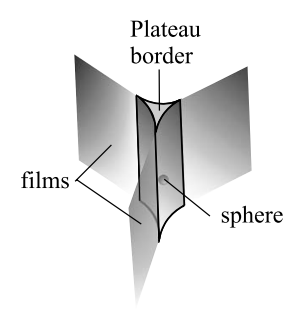

(b)

FIG. 1. (a) Scheme of the setup used to study the settling velocities of solid spheres through fluid channels (not at scale). The channel is obtained by withdrawing a dedicated frame from a reservoir containing a foaming solution. (b) Sketch of the sphere in settling motion in the fluid channel (so-called Plateau border). Note that the cross section of the liquid channel is almost an equilateral triangle except that the three segments joining the vertices are circular arcs.

liquid flow. The particles are first mixed with the foaming solution and placed in a modified three-path gate, ensuring their transfer to the channel holder where they are allowed to settle. Images of the channel during the experiments were grabbed through windows in the cell cover. The successive positions occupied by a sphere along the channel axis are then determined using simple image processing procedures. Another window was also placed at the bottom of the liquid reservoir. In illuminating the channel from the top (through the holder) and using a high speed camera equipped with an appropriate lens, pictures of the channel cross section could be obtained during particle motion. The pictures are then used to determine the particle position within the channel cross section. Unfortunately, we were only able to distinguish between typical positions: particle in a corner [position $x_{2}$ in Fig. 2(a)] at the opposite interface (position $x_{1}$ ) or at the center (position $x_{0}$ ). Note also that the size of the channel holder is far larger than the channel cross section, so that particles are very likely to enter the channel in contact with one or two interfaces. However, we performed a lot of experiments in order to measure the velocity of particles located at the center of the channel. We introduce the parameter $\lambda=d / d_{\text {lim }}$ that compares the size of the particle with the maximum diameter of the circle inscribed in the channel cross section, $d_{\lim }=2 R(2 / \sqrt{3}-1)$, as illustrated in Fig. 2(a). During experiments, $d_{\text {lim }}$ has been kept constant and equal to $140 \pm 10 \mu \mathrm{m}$; the corresponding range of values for $\lambda$ is $[0.2 ; 1]$.

\section{NUMERICAL SIMULATION}

Particulate Reynolds numbers are estimated to be small: $R e \approx 0.1$. The only hydrodynamic force acting on the sphere is thus the viscous drag $F_{D}=3 \pi \mu d V f$, where $f$ is a correction factor due to the presence of the channel walls. The sphere velocity is made dimensionless using the Stokes velocity, $V_{\text {Stokes }}=\left(\rho_{S}-\rho\right) g d^{2} / 18 \mu: \widetilde{V}=V / V_{\text {Stokes }}=1 / f$.

The problem is solved numerically by the finite element method using the commercial software COMSOL MULTIPHYSICS. The dimensionless Stokes equation is used

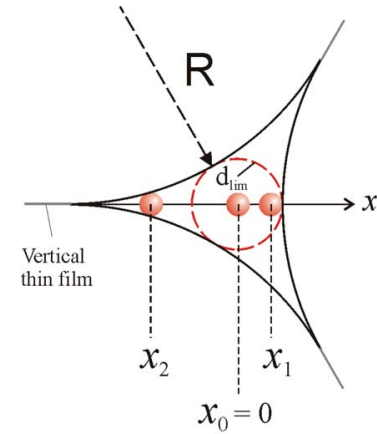

(a)

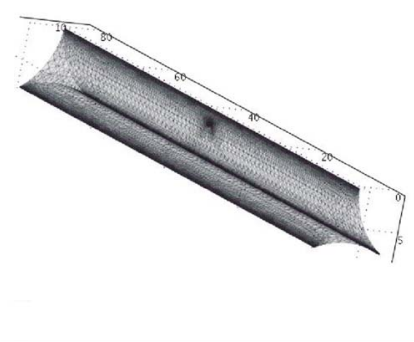

(b)
FIG. 2. (Color online) (a) Channel cross section; the sphere position is determined with the coordinate $x . d_{\text {lim }}$ is the maximum diameter of the circle inscribed in the Plateau border cross section. (b) Example of mesh used to compute the velocity of a sphere in settling motion through the fluid channel (the channel has been inclined for clarity). Note that the mesh is refined near the corners and close to the sphere to properly solve the flow in these regions.

for calculations. The software performs the numerical integration of the stress on the sphere surface, thus providing a value for the drag force for a given dimensionless sphere velocity. Slip condition (free boundary condition) is imposed at the walls of the channel. Zero velocity is imposed at the three corners of every cross section. The viscous stress is set to zero at the channel end in order to obtain a fast decrease in the disturbed flow in this region. In addition, the length of the channel was increased in such a way that obtained results do not depend on the particular value chosen for this study: $L / R=8$. The mesh is refined near the corners and close to the sphere to properly solve the flow in these regions [see Fig. 2(b)]. Calculations are performed with several sphere diameters and corresponding size ratio is within the range $0.1 \leq \lambda \leq 0.99$. The particle position is measured along the median $x$ axis within the cross section [Fig. 2(a)] with the center of the channel as origin, $x_{0}=0$. Coordinates corresponding to the geometrical limits for the sphere inside the cross section are $x_{2}=R\left\{[1+\lambda(2 / \sqrt{3}-1)]^{2}-1\right\}^{1 / 2}-\sqrt{3} R / 3$ and $x_{1}=R[(\sqrt{3}-1)-\lambda(2 / \sqrt{3}-1)]-\sqrt{3} R / 3$. As a limitation of the numerical method, we were not able to perform the calculations for particles in contact with the interface. Thus, a minimum distance was imposed between the sphere surface and the free interface of the channel. As a result, the positions of a sphere in a corner or at the opposite interface correspond to coordinates given by a ratio $\left(x-x_{2}\right) /\left(x_{1}-x_{2}\right)$ equal to 0.01 or 0.99 , respectively.

\section{RESULTS AND DISCUSSION}

Maximum and minimum dimensionless velocities $\left(\tilde{V}_{\max }\right.$ and $\widetilde{V}_{\text {min }}$, respectively) are reported as a function of $\lambda$ in Fig. 3 . Note that the experimental values for the sphere velocity are made dimensionless with the Stokes velocity calculated from the average particle diameter. Although extremely small, the particle polydispersity of the sample thus induces an experimental error. This effect has been found to be the main part of the experimental error and corresponding error bars are presented in Fig. 3. The plot reveals a large 


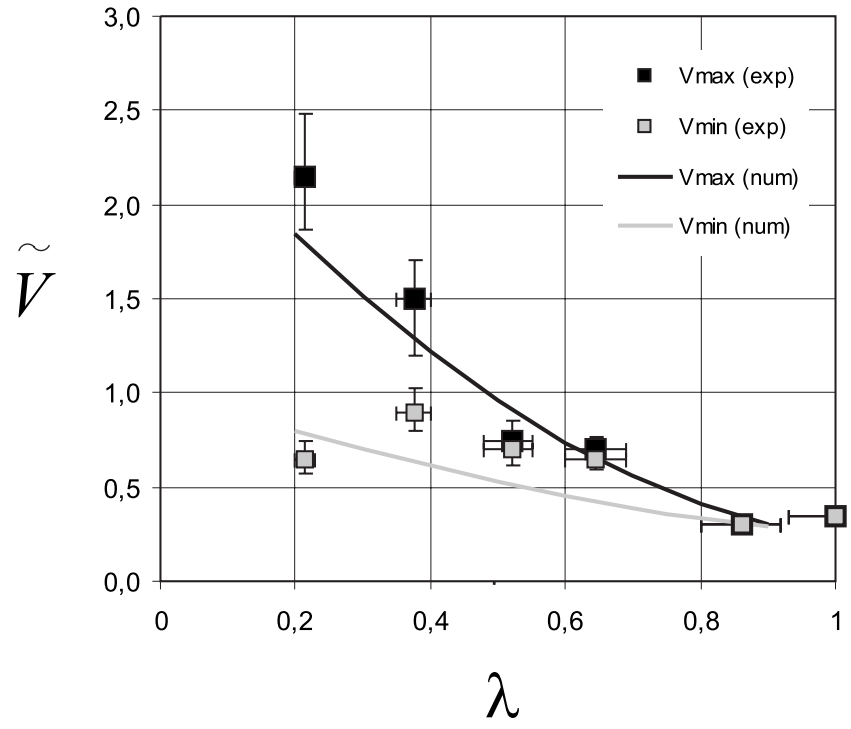

FIG. 3. Experimental values for the maximum and minimum sphere velocities as a function of the size ratio parameter. The numerical values for the maximum and minimum velocities (lines) are presented for comparison.

deviation $\Delta \widetilde{V}=\widetilde{V}_{\max }-\widetilde{V}_{\min }$ for low values of $\lambda$. Moreover, $\widetilde{V}_{\max }$ and $\widetilde{V}_{\min }$ are, respectively, higher and lower than unity. More quantitatively, with respect to the Stokes velocity, the velocity of the sphere in the fluid channel can be reduced by a factor of 0.7-slow motion-as well as increased by a factor of 2-fast motion. It can be seen that $\Delta \widetilde{V}$ is reduced as $\lambda$ increases, and $\widetilde{V}_{\max } \approx \widetilde{V}_{\min }$ for $\lambda \geq 0.8$. The reduction in $\Delta \widetilde{V}$ is accompanied with an overall decrease in the values of the sphere velocity, so that the fast motion is no more observed at high $\lambda$. This transition occurs for the critical value $\lambda_{\text {crit }} \approx 0.5$. In spite of the decrease in the velocity as a function of $\lambda$, it seems that $\widetilde{V}$ does not vanish for $\lambda=1$. Note that for the fluid channel, this geometrical limit does not represent a physical jamming of the sphere inside the channel. Indeed, additional observations have revealed that spheres with a diameter larger than $d_{\text {lim }}$ could also move along the channel. Dimensionless velocities are plotted against the corresponding sphere positions within the channel cross section (Fig. 4) for the smallest size ratio $\lambda=0.2$. Figure 4 provides some understanding of the large deviation observed between $\widetilde{V}_{\max }$ and $\widetilde{V}_{\text {min. }}$. More precisely, it shows up that the velocity is maximum as the sphere locates in the corner of the channel cross section [position $x_{2}$ in Fig. 2(a)] and minimum as it locates in the central area of the cross section (position $x_{0}$ ), whereas intermediate values are obtained for spheres in contact with one interface (position $x_{1}$ ). Thus, the settling velocity increases as the sphere is closer to the walls. A velocity $\widetilde{V} \equiv \widetilde{V}_{2} \approx 2$ is measured as the sphere is in contact with two walls (corner), $\widetilde{V} \equiv \widetilde{V}_{1} \approx 1.2$ when it is in contact with one wall, and $\widetilde{V} \equiv \widetilde{V}_{0} \approx 0.7$ as there is no wall close to the sphere. This experimental result is supported by the numerical curve plotted for comparison in Fig. 4. In particular, the extrapolated numerical value for the sphere in contact with the two interfaces is close to 2 . Figure 5 presents all numerical results as a function of both $x$ and $\lambda$. First of all, fast and slow

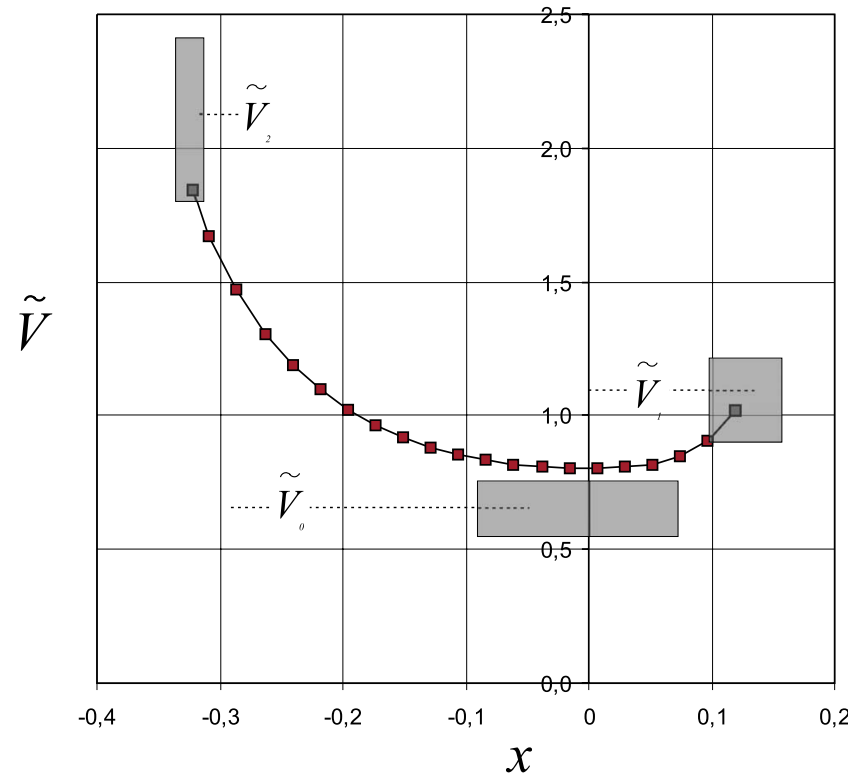

FIG. 4. (Color online) Experimental values for the sphere velocity as a function of the sphere position within the channel cross section for $\lambda=0.2$. Shaded areas account for experimental error. The line represents the corresponding numerical results.

motions are observed. Fast motions are characterized by velocities as high as twice the Stokes velocity when $\lambda<0.2$. For $\lambda=0.2$, the numerical value for the velocity of spheres located at $x_{2}$ is close to $\widetilde{V}_{\max }$. Actually, this good agreement is found for every value of the size ratio, as observed in Fig. 3. Moreover, the numerical value for the deviations $\tilde{V}\left(x_{2}\right)-\tilde{V}\left(x_{0}\right)$ is found to decrease as $\lambda$ increases from 1.36 for $\lambda=0.1$ to 0.017 for $\lambda=0.9$. The sphere velocity $\tilde{V}\left(x_{0}\right)$ decreases from the expected Stokes velocity as $\lambda \rightarrow 0$ to a nonvanishing value, in strong contrast to channels with solid

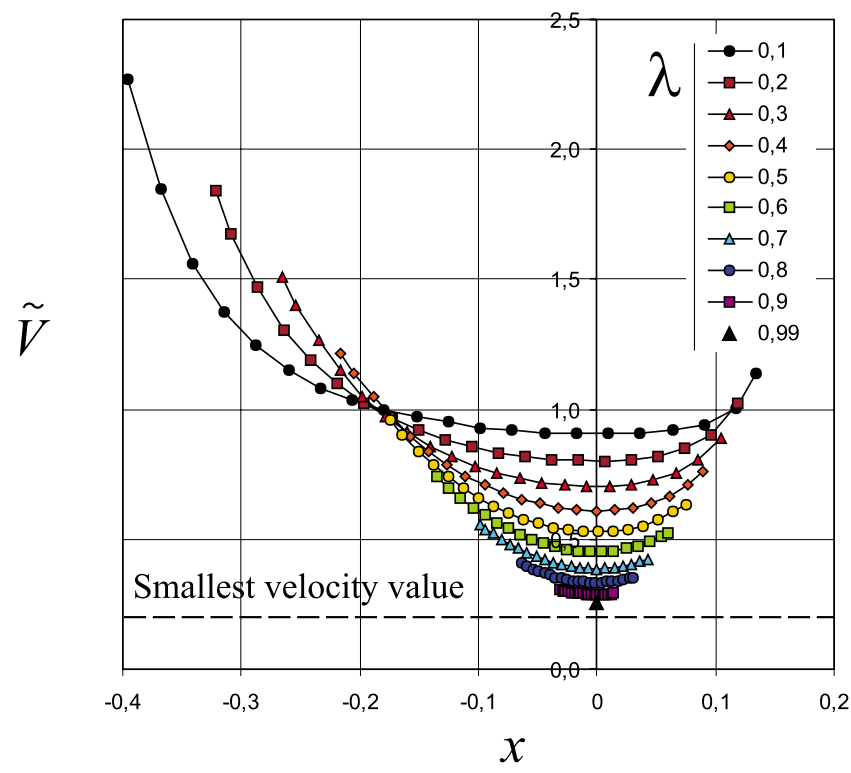

FIG. 5. (Color online) Numerical values obtained for the sphere velocity as a function of the coordinate and for several values of the size ratio within the range of $0.1-0.99$. The dashed-line represented the smallest dimensionless velocity for a sphere in a fluid channel $\widetilde{V}=0.25$. 

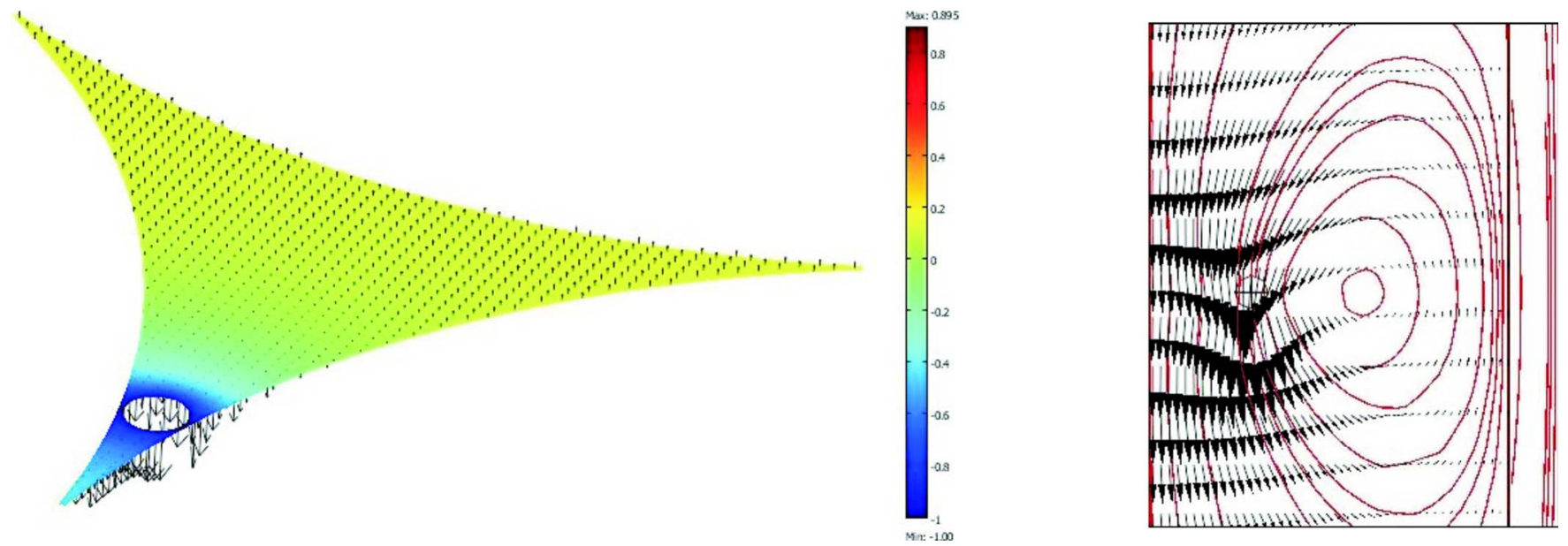

FIG. 6. (Color online) Flow induced by the motion of a sphere along the fluid channel and located at position $x_{2}$ [see Fig. 2(a)] for $\lambda=0.19$. (Left) Velocity field (the arrows) and the vertical velocity (colors/shades) within the channel cross section passing through the center of the sphere. (Right) Velocity field and streamlines within a vertical median slice along $x$ axis.

walls. The value obtained at $\lambda=0.99$ is 0.2547 , and the extrapolated value at $\lambda=1$ is very close to 0.25 . This result is in agreement with the corresponding experimental value: $\widetilde{V}_{\text {max }} \approx \widetilde{V}_{\text {min }} \approx 0.3$ (see Fig. 3). This effect can be attributed (i) to the geometry of the channel cross section, enabling the liquid backflow to proceed through the corners, and (ii) to the interface mobility, allowing the movement to proceed even as the sphere is touching the three confining interfaces. On the other hand, at small $\lambda$, the motion of the sphere is only weakly influenced by the nature of the wall. For $\lambda=0.2$, the numerical value $\tilde{V}\left(x_{0}\right)=0.79$ compares well with the experimental value (see Fig. 4). This value is also close to that published for a sphere in settling motion along the axis of a vertical tube with rigid walls, whatever the geometry of the cross section: cylindrical ${ }^{6,7}$ or triangular. ${ }^{6}$ Moreover, a numerical calculation with the present cross-section geometry and no-slip boundary condition provided a value slightly below $0.7 .^{15}$ Both experimental and numerical values of the velocity for a settling sphere close to one wall of the channel are $\widetilde{V}_{1} \approx 1.1-1.2$. In Refs. $16-18$ the settling velocity of a particle close to a planar free interface was calculated and found equal to $\widetilde{V}_{\text {plane }} \approx 1.36$. The observed deviation between $\widetilde{V}_{\text {plane }}$ and $\widetilde{V}_{1}$ can be attributed to backflow experienced by the sphere in the three-dimensional confined geometry. As the backflow is amplified when $\lambda$ increases, the deviation between $\widetilde{V}_{\text {plane }}$ and $\widetilde{V}_{1}$ increases as $\lambda$ increases. A similar discussion can be made about $\widetilde{V}_{2}$. The main difference lies in the value itself, which reflects the influence of the two interfaces confining the sphere in the corner. A comparison can be made to a previous numerical study of sphere motions confined between two fluid walls. ${ }^{19}$ Their calculations predicted that for sufficiently low values of the surface viscosity, an efficient fast motion regime can be obtained. The reported values are fully consistent with $\widetilde{V}_{2}$, although in the two plane configuration, the slow motion regime is not
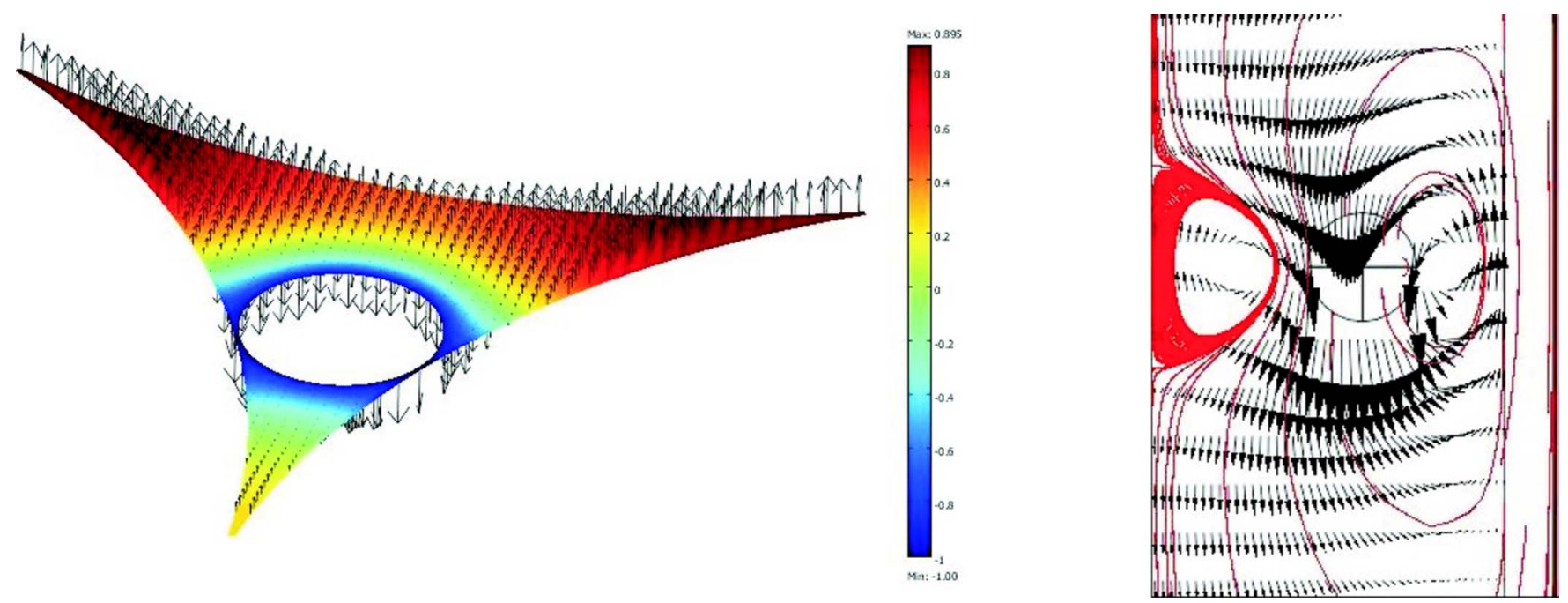

FIG. 7. (Color online) Flow induced by the motion of a sphere along the fluid channel and located at position $x_{2}$ [see Fig. 2(a)] for $\lambda=0.65$. (Left) Velocity field (the arrows) and the vertical velocity (colors/shades) within the channel cross section passing through the center of the sphere. (Right) Velocity field and streamlines within a vertical median slice along $x$ axis. 
observed because the sphere is confined only along one direction. Actually, the slow motions observed with the present geometry result from the drag effect associated with the backflow as $\lambda$ increases. As already said, the backflow effect is all the more pronounced as the size ratio is high, so that a critical value can be identified above which only slow motions are observed. Figure 5 provides a numerical evaluation for this critical value: $\lambda_{\text {crit }}=0.5$. This value is in very good agreement with the experimental one. Thus, at high size ratio, the backflow effect appears to be responsible for the increase in the drag force in the fluid channel. This is in contrast to channels with rigid walls, where the shearing of the liquid layer between the sphere and the closest interface becomes the dominant effect at high size ratio. Figures 6 and 7 illustrate the effect of the backflow in the present situation. For $\lambda=0.19$, the backflow takes place in the large free area with the cross section. For $\lambda=0.65$, the free area is significantly decreased so that the effect of the backflow and the associated drag force are more pronounced. In this respect, note the difference for the flow within the corner area: the liquid velocity is downward for $\lambda=0.19$, whereas it is upward for $\lambda=0.65$. This transition as a function of $\lambda$ constitutes a major difference with previously reported laws of motion in wall-confined liquids.

\section{CONCLUSION}

New experimental and numerical results have been presented for the motion of solid particles in confined geometries with free surfaces. It has been shown that in such geometries, the deviation from the classical nonslip boundary condition at the confining wall induces a nontrivial behavior for the particle drag coefficient. For sufficiently small particles, slow and fast motions are observed for the particle along the channel, depending on the particle position within the channel cross section and the sphere/channel size ratio $\lambda$. The velocity corresponding to fast motions can be as high as twice the Stokes velocity when $\lambda<0.2$. Moreover, the fast motions are not observed anymore when the size ratio exceeds a critical value $\lambda=0.5$. As another major difference with the solid wall channel, for $\lambda>0.5$ the sphere velocity is reduced only due to the backflow effect. As an interesting result, the sphere velocity is never smaller than $\frac{1}{4}$ of the Stokes velocity, even for a size ratio equal to unity.

\section{ACKNOWLEDGMENTS}

We gratefully acknowledge the financial support from the Agence Nationale de la Recherche (Grant No. ANR-05-JCJC-0234-01), the E.S.A. (MAP No. A099-108: C14914/02/NL/SH), and the French Space Agency (Convention No. CNES/70980).

${ }^{1}$ G. G. Stokes, "On the effect of the internal friction of fluids on the motion of pendulums," Trans. Cambridge Philos. Soc. 9, 8 (1851).

${ }^{2}$ J. Happel and H. Brenner, Low Reynolds Number Hydrodynamics, 2nd ed. (Noordhoof, Leiden, 1973).

${ }^{3}$ A. J. Goldman, R. G. Cox, and H. Brenner, "Slow viscous motion of a sphere parallel to a plane wall. I. Motion through a quiescent fluid," Chem. Eng. Sci. 22, 637 (1967).

${ }^{4}$ M. E. Staben, A. Z. Zinchenko, and R. H. Davis, "Motion of a particle between two parallel plane walls in low-Reynolds-number Poiseuille flow," Phys. Fluids 15, 1711 (2003); erratum, ibid. 16, 4206 (2004).

${ }^{5}$ M. E. Staben and R. H. Davis, "Particle transport in Poiseuille flow in narrow channels," Int. J. Multiphase Flow 31, 529 (2005).

${ }^{6}$ A. Miyamura, S. Iwasaki, and T. Ishii, "Experimental wall correction factors of single solid spheres in triangular and square cylinders and parallel plates," Int. J. Multiphase Flow 7, 41 (1981).

${ }^{7}$ R. P. Chhabra, "Wall effect on free-settling velocity of nonspherical particles in viscous media in cylindrical tubes," Powder Technol. 85, 83 (1995).

${ }^{8}$ J. Ou, B. Perot, and J. P. Rothstein, "Laminar drag reduction in microchannels using ultrahydrophobic surfaces," Phys. Fluids 16, 4635 (2004).

${ }^{9}$ J. Hyväluoma and J. Harting, "Slip flow over structured surfaces with entrapped microbubbles," Phys. Rev. Lett. 100, 246001 (2008).

${ }^{10}$ S. A. Koehler, S. Hilgenfeldt, E. R. Weeks, and H. A. Stone, "Drainage of single Plateau borders: Direct observation of rigid and mobile interfaces," Phys. Rev. E 66, 040601 (2002).

${ }^{11}$ O. Pitois, C. Fritz, and M. Vignes-Adler, "Liquid drainage through aqueous foam: Study of the flow on the bubble scale," J. Colloid Interface Sci. 282, 458 (2005)

${ }^{12}$ J. Sur and H. K. Pak, "Capillary force on colloidal particles in a freely suspended liquid thin film," Phys. Rev. Lett. 86, 4326 (2001).

${ }^{13}$ H. A. Stone, A. D. Stroock, and A. Ajdari, "Engineering flows in small devices: Microfluidics toward a lab-on-a-chip," Annu. Rev. Fluid Mech. 36, 381 (2004).

${ }^{14}$ S. J. Neethling and J. J. Cilliers, "Modeling flotation froths," Int. J. Min. Process. 72, 267 (2003).

${ }^{15} \mathrm{~F}$. Rouyer, private communication (2009).

${ }^{16}$ S. H. Lee and L. G. Leal, "Motion of a sphere in the presence of a plane interface. Part II. An exact solution in bipolar coordinates," J. Fluid Mech. 98, 193 (1980).

${ }^{17}$ K. D. Danov, R. Aust, F. Durst, and U. Lange, "Slow motions of a solid spherical particle close to a viscous interface," Int. J. Multiphase Flow 21, 1169 (1995).

${ }^{18}$ A. V. Nguyen and G. M. Evans, "Movement of fine particles on an air bubble surface studied using high-speed video microscopy," J. Colloid Interface Sci. 273, 262 (2004).

${ }^{19}$ K. D. Danov, R. Aust, F. Durst, and U. Lange, "Influence of the surface viscosity on the drag and torque coefficients of a solid particle in a thin liquid layer," Chem. Eng. Sci. 50, 263 (1995). 